\title{
Short Video Strategy Analysis from the Self-Media Industry-Taking ByteDance Company as an Example
}

\author{
Qixiang Sun* \\ Zhejiang University of Finance \& Economics Dongfang College, Haining 314400, China. E-mail: 860499567@qq.com
}

\begin{abstract}
In today's smart Internet era, self-media as an emerging industry uses short video to affect people in many ways. In perspective of short video, this article takes ByteDance company as an example to analyze the status of short video mode combined with its own advantages and disadvantages, and it will further study the problems in the development of self-media. At the same time, it puts forward corresponding strategies to provide a reference for corporate strategy formulation.
\end{abstract}

Keywords: Self-Media Industry; Douyin Short Video; ByteDance Company Strategy

\section{Introduction}

In recent years, with the rapid development of smart phones and mobile Internet, many media platforms have emerged, such as Weibo, Toutiao, Douban, and Douyin App. These emerging media are collectively referred to as self-media ${ }^{[1]}$. Among them, the most striking is Douyin, which just won the title of the largest short video platform in China at the beginning of this year. According to the latest 2019 Douyin data report, as of January 6, 2020, the number of active users on Douyin day has exceeded 400 million, and it has become the contemporary majority of people's leisure mainstream entertainment. This new form of media is not only a way. As a force on the Internet platform, it has changed the way people accepting entertainment, leading the trend of the new era, and the ByteDance behind it is also known by more people. Since established in 2012, it has not only quickly established a foothold, but also broken through the monopoly of BAT (Baidu, Alibaba and Tencent ${ }^{[2]}$. Today, it has become a new generation of Internet giants in artificial intelligence and big data, which are closely related to ByteDance's unique business vision and the right strategic direction.

Taking the development of ByteDance's strategy as an example, this article analyzes the advantages and disadvantages of the short video model and the social impact, valuable business experience and future development of the multi-field strategic cooperation mode.

\section{ByteDance company profile}

Beijing ByteDance Co. LTD. (hereinafter referred to as ByteDance) was on the line in 2012, and it is one of the earliest technology enterprises that apply artificial intelligence technology to mobile Internet scene in China. This company's main business are Internet technology development, technology promotion and transfer and other services. Its products such as Toutiao, Douyin, and Watermelon Video now have market shares. The company has conducted in-depth exploration of long-term and open issues in artificial intelligence, big data and other related fields to help it

Copyright (C) 2020 Qixiang Sun

doi: $10.18686 / \mathrm{mcs} . \mathrm{v} 2 \mathrm{i} 3.1327$

This is an open-access article distributed under the terms of the Creative Commons Attribution Non-Commercial License

(http://creativecommons.org/licenses/by-nc/4.0/), which permits unrestricted non-commercial use, distribution, and reproduction in any medium,

provided the original work is properly cited. 
realize its vision for future development. Through massive information collection, big data mining and user behavior analysis, its independently developed Douyin App intelligently recommend personalized information for users, thus creating a brand new short video transmission mode.

\section{Analysis of the current status of short video development from an enter- prise perspective}

\subsection{Rich content, wide coverage, but the "repetitive" problem}

The biggest highlight of the short video mode is that its knowledge covers a wide range. In the information society, what people need most is to obtain convenient and rich content. On the one hand, the reason is that the traditional media model has a very slow text propagation speed and lacks access to the channels ${ }^{[3]}$. On the other hand, some high-quality content is not easy to be discovered, and it is naturally difficult for people to see it. However, the emergence of short video just to fill the vacancy, as of December 8, 2018, Dinyin on fans had knowledge category creator million for nearly 18,000 , over 3 million short videos of knowledge type have been published in total ${ }^{[3]}$, and the uninterrupted push can provide users with rich choices.

Although rich in short video content, there are more serious problems of repetition and heat transfer. When a novel short video goes viral, it will trigger a series of similar repetitive content, similar to "fashion imitation show" and "funny paragraph". Meanwhile, Douyin's unique intelligent calculation mechanism will continue to push users to browse of similar content, it is easy to put the repeat of the content again pushed to the user, reducing the sense of user experience. There are also the resulting "video plagiarism", and "heat" problem is also common ${ }^{[3]}$. Higher playback volume the short video is full of unrelated topics such as "pet familiar everyday", "advertising promotion", and some of the infringement issues will also cause unnecessary disputes and reduce the credibility of the online platform.

\section{2 "Flow trap" among wide and gradually popular audience}

Nowadays, much short video software on the market has broken through the time and space limitations. Only one mobile phone can achieve "zero distance" contact with big coffee, Internet celebrities, and even well-known businesses. Many media industries have also come, such as "well-known online celebrity e-commerce bring goods", "home-made online red hot sauce", "small movies" of popular science institutions, etc. The main reason is that its popular characteristics allow everyone to choose the right to watch this type of video, many of them on weekdays not well known self-media has gradually entered the public's view. In addition, the value of the popular class has gradually been discovered by short video platforms and related researchers. In addition, the value of the mass class itself has been gradually discovered by the short video platforms and relevant researchers, and the power of many we-media and merchants has been decentralized, realizing real participation and interaction.

Every advantage has its disadvantage. When everyone can participate in the process of video dissemination, the public lacks the necessary sense of responsibility and media literacy for video dissemination, coupled with weak platform supervision. In order to earn traffic money and blindly increase user participation rate, many "we media" often spread some short videos with only gimmicks but no real content, such as Douyin often have "mysterious underwater world", "AI will eventually replace humans", "99\% of people do not know the secret". This type of video uses past videos or photos to deceive the user's attention and disseminate inappropriate comments. At the same time, the phenomenon of plagiarism among some Internet celebrities and the proliferation of works is also common. They often use eye-catching topics to attract customers and use popularity to get attention, but there is no actual content, in order to cheat the attention of advertisers. It is difficult to effectively advertise and affect the interests of users and businesses.

\subsection{User traffic obtained and barriers that are difficult to break through}

Today's Douyin, Toutiao, Volcano Small Video and other software have won the favor of most users in first-, second-, and even third-tier cities. Currently, Douyin DAU (number of daily active users) exceeds 250 million, far sur- 
passing the same period of Quick Hands. In terms of product traffic or user time, ByteDance is second only to Tencent, close to Alibaba, and it has surpassed Baidu. In the information age, it means that there is flow of profit, according to Douyin short video platform statistics. The revenue of Douyin has reached 20 billion yuan in 2018, and the main revenue comes from information flow advertising and open screen advertising, but if companies want to go further and break through the traffic ceiling, in today's Wolf Ring in the Internet market further, it is difficult in the traditional competition track. As there are, in order to avoid the layers of siege Internet giants continue to optimize its own brand and strengthen its technical difficulty. On the one hand, smart devices are changing rapidly, and new products and technologies are emerging one after another, as appropriate innovation is inevitable. On the other hand, it is now the era of rapid innovation of Internet technology. Big data, cloud computing, AI and other core Internet technologies are developing rapidly. The one that can truly master the most cutting-edge innovative technology will laugh to the end in the product competition.

ByteDance past strategy was to use today's headline content information to develop new areas. It mastered user traffic and content orientation with searching and algorithm recommendations to "fast trial and error" in multiple directions, which compressed costs to develop multiple products. They can always find the appropriate APP and focus on training. Small videos like Douyin and Volcano are developed in this way, but their own hard technology is limited by many smart push vulnerabilities, and it is difficult to develop hardware products, affecting the development of the enterprise.

\subsection{Going-out strategy on the basis of not strict management review}

During the past two years, the development of short video industry was gradually approaching the industry ceiling, so the short video software led by Douyin began to focus on the international layout strategy. At present, TikTok (overseas version of Douyin) musically cover more than 150 countries and regions globally. In 2018, TikTok reached 119 million downloads in India, 39.6 million in the United States, and 28.4 million in Turkey and 24.3 million in Russia. But there are also inevitable risks in rapid development and expansion. Short video internal audit is not strict, and there is a lot of vulgar content. In 2019, Indonesia unilateral embargo Douyin account. In the same year, the United States fined tens of millions of dollars for objectionable juvenile content ${ }^{[4]}$. In addition, there are many adverse realities, such as the difficulty of traditional management mode, the risk of network security, the risk of information authenticity and so on. How to strengthen the control and governance of self-media to reduce the risks and negative impacts of short videos on society is a topic of the era ${ }^{[5]}$.

\section{Enterprise development strategy and forecast in the new era}

\subsection{Breaking through in the field of Internet innovation}

In a dance competition, the more technically difficult a dancer is, the higher the score he or she is likely to get without making a mistake. ByteDance also needs to continue to increase its own technical difficulty ${ }^{[4]}$. At this stage, the core business revenue of ByteDance mainly comes from the short board of advertising. Enterprises should increase human and financial investment in the artificial intelligence industry and continue to build "machine learning, large data mining, cloud computing, building accurate and efficient content distribution" for the organization mode; to improve big data recommendation algorithm for the push vulnerabilities; to form a previous simple batch push relevant news; to accurately locate users to push content they are interested in; to truly realize the "different subjects from different angles" of content distribution. At the same time, patent acquisition is also one aspect of technological innovation. Since 2018, ByteDance has been awarded by China and the United States and other national patent offices. Except for many patents of inventions and designs inside, the company's direct procuring patents from outside are also magic weapons to help their development. In January 2019, ByteDance acquired some of the patent rights of Smartisan, and new technology can continue to explore new areas. It can save the cost and time of R \& D and innovation and directly obtain the patent right of the new production ${ }^{[2]}$. 


\subsection{Multi-field and cross-platform cooperation}

The cross-platform cooperation under the short video mode started in 2019. The biggest scale is the online celebrity live broadcast, the form of e-commerce with goods. The convenient live broadcast platform and the influence of millions of fans have attracted a large number of e-commerce owners to cooperate, such as Tmall, Taobao, JD and the evolving personal brands of Internet celebrities. In the next few years, ByteDance will continue to expand its cooperation with major offline online stores and use the well-established popularity to attract traffic; meanwhile, in order to meet the needs of users of different ages, they can work with various authorities and universities cooperation, such as inviting experts and professors to conduct courses online and offline, so that all kinds of cultural knowledge can be presented to the public in the most gentle way. At the same time, it can also strengthen the internal learning of the enterprise to improve the cultural quality and stimulate the interest and ability of employees.

In addition to this model of encouraging education, ByteDance will continue to explore the field of education, because now high-quality education has become the goal pursued by every family. When an enterprise focuses on technological innovation and technical services, technology and education combination is an inevitable trend. In recent years, ByteDance has hatched products such as Gogokid and AIKID, and acquired Xuebajun App ${ }^{[6]}$, which has accumulated rich experience in the field of early childhood and adolescent education. Next, ByteDance wants to consider the consumer's point demand, such as the launch of online one-on-one tutoring for young people, or partner with a smart phone company to launch a truly both learning and entertainment of intelligent machines. The market potential of the product needs to be further developed.

\subsection{Optimization of the internal management model and improvement of its own risk man- agement plan}

As a huge enterprise in a certain scale, perfect internal management organization is essential. In recent years, the Chinese government always attached importance to the construction and management of the Internet, the "active use, scientific development, according to management, to ensure the security" approach is proposed, and the "Internet Management Regulations People's Republic of China" and other laws and regulations are introduced ${ }^{[7]}$. However, in recent years, the number of times of chaotic management and inadequate audits in the media industry has remained relatively high. In the final analysis, the management of the company's internal platform cannot be supervised by the outside world. Some positions have appeared "multiple jobs". In response to the above risks, comprehensive considerations suggest to govern from the following aspects:

First, from the legal level, enterprises should strictly improve the enforcement of laws and regulations from top to bottom and further elaborate on the implementation of specific management regulations for various types of management regulations. They need to truly achieve "accountability to people" without avoiding. Do not pass the buck.

Second, the management level should improve the technical management and control capabilities of each software platform. Today's platforms such as Toutiao, Douyin, and Watermelon Video have the prosecution and other functions. Most short video forums have also set up keyword technical review and other means, but there is also a need for a complete set of information management and control technologies. Using these technologies to automatically filter and delete reducing and reporting sensitive speech and inappropriate harmful content can not only save labor costs, but also protect citizens' freedom of expression. It also ensures the security of the network environment and promotes the positive energy of society. What's more, strengthening network security construction and protecting information security on self-media platforms are also essential ${ }^{[8]}$.

Third, in response to the illegal and non-compliant marketing activities of Internet celebrities that has great influence in each live broadcast platform when operating their own brands, the internal rectifications of each platform should bear the brunt. The rectifications include forcibly banning their "marketing number" and "privacy" "account", training them in relevant marketing education, guiding them to take the lead in exemplary role, improving legal awareness, and resisting unhealthy practices. For some minors, organizations or individuals of college students, we must strengthen the education and popularization of marketing knowledge of we media. The popularization of education in 
media marketing knowledge allows them to improve their ability to distinguish between right and wrong, to consume rationally, and not to blindly follow suit.

Fourth, enterprises should set up a long-term early warning mechanism, the main body that strengthens the role of the content "gatekeeper" and integrates this mechanism into the daily management mode of the enterprise; It is also necessary to strengthen the connection between the industry platform and the government and enterprises to ensure that the governance function has been implemented. Of course, the close communication and coordination between the various departments of the enterprise is a bridge. There is no close connection between the departments, and everything is empty talk.

\section{Conclusion}

The beautiful dances in the world are extremely difficult, and excellent dancers have to pay unimaginable hardships ${ }^{[9]}$. In this self-media market full of uncertain factors, the ByteDance exerts its own advantages with effective risk avoiding and innovative products. Just like a beautiful dancer, it tries its best to stretch dance posture in the emerging industry of self-media, even Internet giants are always on the prowl, but only by defeating these, can the ByteDance jumps out of the beautiful dance steps and become more powerful.

\section{References}

1. Gao S. Analysis of the advantages and disadvantages of self-media marketing under the background of "Internet+" (in Chinese). Marketing Management Review 2020; (1): 140.

2. Fang Z, Mi X. "Super Unicorn" byte-beating non-R\&D innovation (in Chinese). Enterprise Reform and Management 2019; (9): 194+203.

3. Guo K. Analysis on the scientific communication mechanism of short video platform from the perspective of value co-creation-Take Douyin short video platform as an example (in Chinese). Southeast Communication 2020; (2): 31-33.

4. Jian D. Byte beat, heart beat (in Chinese). China Internet Week 2019; (7): 20-21.

5. Feng G. How to act as a "gatekeeper" in the fission of self-media communication? (in Chinese). Foshan Daily 2020; (A06).

6. Zuo P. Can byte-beating mobile phone dreams come true? (in Chinese). Science and Technology Daily 2019; (5).

7. Zhang Z. Seeing operators' cross-border new media from the strategic cooperation between China Mobile and ByteDance (in Chinese). C-Enterprise Management 2019; (3): 12-15.

8. Jiang L, Xu Y, Yan X. Analysis of the current self-media chaos and its governance path (in Chinese). Journalism Lover 2019; (1): 8-13.

9. Sun H. Five trends in the development of my country's new media industry (in Chinese). Young Journalist 2019; (19): 46. 